\title{
Ordered Attributed Grammars
}

\section{Uwe Kastens}

Institut für Informatik II, Universität Karlsruhe, Postfach 6380,

D-7500 Karlsruhe 1, Germany (Fed. Rep.)

Summary. Ordered attributed grammars are defined as a large subclass of semantically well-defined attributed grammars proposed by Knuth. An attributed grammar is ordered if for each symbol a partial order over the associated attributes can be given, such that in any context of the symbol the attributes are evaluable in an order which includes that partial order. The definition does not refer to a predefined strategy for attribute evaluation, e.g. several passes from left to right. For each attributed grammar evaluable by any predefined evaluation strategy such an order exists. The ordering property can be checked by an algorithm, which depends polynomially in time on the size of the input grammar. "Visit-sequences" are computed from the attribute dependencies given by an ordered attributed grammar. They describe the control flow of an algorithm for attribute evaluation which can be part of an automatically generated compiler.

\section{Contents}

1. Introduction . . . . . . . . . . . . . . . . . . . . . . 230

2. Attributed Grammars. . . . . . . . . . . . . . . . . . . . . . . . . . . . . 235

3. Deciding Whether Attributed Grammars are Ordered . . . . . . . . . . . . . . . . . . . . . . . 242

4. Hierarchical Classification of Attributed Grammars . . . . . . . . . . . . . . . . . . . . . . . 244

5. Visit-Sequences for Ordeted Attributed Grammars . . . . . . . . . . . . . . . 247

6. Implementations of Visit-Sequences . . . . . . . . . . . . . . . . . 251

7. An Algorithm for Computing Visit-Sequences . . . . . . . . . . . . . . . . . . . 255

8. Conclusion . . . . . . . . . . . . . . . . . . . . . . . . . . . . . 256

9. References

\section{Introduction}

Attributed grammars (AGs) are a well suited means for a complete definition of a programming language, including all statically determinable properties of the language. In $[9,10]$ Knuth established a condition for well-defined attributed 
grammars (WAGs): The semantic rules of an AG are well-defined if and only if there is no sentence of the language with circularly dependent attributes. In [4] it was proven that the decision whether an $A G$ is well-defined is an intrinsical exponential problem.

In this paper we introduce "ordered attributed grammars" (OAGs) as a subclass of WAGs. Grammars of this class are characterized by the following condition: For each symbol of the grammar a partial order over the associated attributes can be defined, such that in any context of the symbol the attributes are evaluable in that order. Such grammars have several desirable properties with respect to the definition of programming languages and to automatic generation of compilers:

- An OAG is well-defined in the sense of [9].

- The class of OAGs is defined without any assumption on the strategy for attribute evaluation, e.g. one or more passes over the program from left to right as for example in [1]. Furthermore for all AGs of a class based on predefined evaluation strategies such an order exists.

- For an OAG one can automatically construct compiler algorithms evaluating the attributes of any sentence of the language.

- The class of OAGs is sufficiently large for programming language definition. The context dependencies of programming languages can usually be defined by OAGs. The dependencies between the properties (attributes) of a language element define a partial order in each possible context. In general the superposition of these partial orders result in a new partial or linear order. In this sense the condition for the subclass is natural

- It is decidable whether an $A G$ is ordered. The time needed for that decision and for the computation of the order depends polynomially on the input grammar. The analysis of OAGs for usual programming languages can be done in reasonably short time.

Compiler generating systems based on different subclasses of AGs already exist ( $[2,11,12,14])$. In order to generate complete compilers the AGs are extended such that they describe the code to be generated for a target machine. (Different approaches are discussed in $[6,16,17]$.) A generator based on OAGs $(G A G)$ is currently being implemented by the author. A rather complete bibliography on AGs is found in [15].

In Sect. 2 the notation of AGs and an example is given, which is referred to in further sections. Section 3 defines the condition for OAGs and how to check it. The position of OAGs within the hierarchy of classes of AGs is shown in Sect. 4. The ordering property of an OAG is used in Sect. 5 for computing tree walk rules (called "visit-sequences") for attribute evaluation. They can be implemented in automatically generated compilers as shown in Sect. 6. In Sect. 7 we give an abstract algorithm for checking whether an $A G$ is ordered and computing the visit-sequences and discuss its complexity.

\section{Attributed Grammars}

In this section we introduce our notation for AGs. It differs to some degree from other notations used in literature in order to achieve completeness and readability. 
As an example for further reference we consider the definition of a small expression language.

An $\mathrm{AG}$ is based on a context-free grammar which is augmented by attributes, functions defining values of attributes, and conditions over attributes. Each attribute describes a property of a language element, which is defined by a function in a context dependent manner. The conditions restrict the combinations of these properties according to static semantics.

An $A G$ is defined by a 5 -tupel

$$
A G=(G, A, V A L, S F, S C) \text {. }
$$

$G=(N, T, S, P)$ is a reduced context-free grammar, where $N$ is the set of nonterminal symbols, $T$ is the set of terminal symbols, $V=N \vee V$ is the vocabulary of the grammar, $S \in N$ is the start symbol, and $P$ is the set of syntactic rules. Each syntactic rule $p \in P$ has the form

$$
p=X_{0}: X_{1} \ldots X_{n p}, \quad n p \geqq 0
$$

$X_{i}$ denotes an occurrence of a symbol of $N$ for $i=0$ and of $V$ for $i>0$. In the following $X_{i}$ will always denote an occurrence of a symbol $X$ in such a rule $p$. The qualification of the index $0 \leqq i \leqq n p$ will be implied.

$A$ is a set of attributes. Each attribute is associated to exactly one symbol $X \in V . A_{X}$ is the set of attributes associated to $X$. The elements of $A_{X}$ are denoted $X \cdot a, X \cdot b, \ldots$. We have

$$
A=\bigcup_{X \in V} A_{X} \text { and } A_{X} \wedge A_{Y} \neq \emptyset \text { implies } X=Y \text {. }
$$

$A$ is partitioned into two disjoint subsets $A I$ and $A S$, the inherited and the synthesized attributes:

$$
A=A I \vee A S \text { and } A S \wedge A I=\emptyset \text {. }
$$

Hence each $A_{X}$ is partitioned into two subsets $A I_{X}$ and $A S_{X}$. For each occurrence $X_{i}$ of a symbol $X$ in a rule $p$ there is an attribute occurrence $X_{i} \cdot a$ for all attributes $a \in A_{X}$.

$$
A_{p}=\bigcup_{i=0}^{n p} \bigcup_{a \in A X} X_{i} \cdot a
$$

is the set of all attribute occurrences in $p$.

$$
S F=\bigcup_{p \in P} S F_{p}
$$

is a set of semantic functions associated to rules $p \in P$. Each semantic function defines the value of an attribute occurrence in $p$ depending on $k \geqq 0$ attribute occurrences in $p$ :

$$
\begin{aligned}
& f \in S F_{p} \\
& f \subseteq \underset{j=0}{X} \operatorname{DOM}\left(b_{j} \in A_{p}\right) \rightarrow \operatorname{DOM}\left(a \in A_{p}\right), \quad \text { for a } k \geqq 0 \\
& \text { e.g. } X_{i} \cdot a:=g\left(X_{m} \cdot c, \ldots, X_{n} \cdot d, \ldots\right) .
\end{aligned}
$$


The values defined for the attribute occurrences are taken from a set of possible values, the domain of the attributes: $\operatorname{DOM}\left(X_{i} \cdot a\right)=\operatorname{DOM}(X \cdot a)$, for $X=X_{i}$.

$V A L$ is the set of the domains of all attribute values:

$$
V A L=\{\operatorname{DOM}(a) \mid a \in A\} .
$$

For each $X_{i} \cdot a$ of the set of defining occurrences

$$
A F_{p}=\left\{X_{i} \cdot a \mid(i=0 \text { and } a \in A S) \text { or }(i>0 \text { and } a \in A I)\right\}
$$

there is exactly one function in $S F_{p}$ defining the value of $X_{i} \cdot a$. Thus it is ensured that the value of each attribute is determined uniquely in any context. The set of applied occurrences (not defined by $S F_{p}$ ) is

$$
A C_{p}=A_{p} \backslash A F_{p} .
$$

The attribute values are statically defined by the semantic functions, comparable to the objects and functions of the lambda calculus. The functions must not be looked upon as algorithms on variable attributes!

Let $s$ be a sentence of $L(G)$ which is derived by

$$
S \Rightarrow u Y y \rightarrow_{q} u v X x y \rightarrow_{r} u v w x y \Rightarrow S .
$$

A node $K$ representing the symbol $X$ in the structure tree for $s$ is called an instance of $X$ denoted by $K_{X}$. For each attribute $X \cdot a$ an attribute instance $K_{X} \cdot a$ is associated to $K_{X}$. The values of the inherited attribute instances of $K_{X}$ are defined by functions in $S F_{q}$, the values of the synthesized ones by functions in $S F_{r}$. A structure tree augmented by the attribute instances is called an attributed structure tree.

$S C$ is a set of semantic conditions, one associated to each rule $p$ :

$$
S C_{p} \in \underset{j=1}{\gtrless} \operatorname{DOM}\left(a_{j} \in A_{p}\right) \rightarrow\{\text { true, false }\}, \text { for a } k>0 \text {. }
$$

A sentence $s \in L(G)$ is a sentence of the language $L(A G)$ iff for each application of a rule $p$ in the derivation of $s$ the values of the corresponding attribute instances meet the condition $S C_{p}$. The following discussions are restricted to attribute dependencies determining the evaluation order. As semantic conditions and the domains of attribute values do not influence the attribute dependencies they are not considered here. In an actual language definition or compiler generation domains correspond to data types of modern high level programming languages. (For more details see [7].)

As an example for further reference we consider the definition of a simple expression language. The example covers four of the most important context sensitive properties of languages: scope rules, mode checking, coercion and operator identification. In addition an optimizing method (constant folding) is incorporated in a very simple way. We use an informal notation which can be converted into a systematic description language [7]. 
Ordered Attributed Grammars

The following attributes are used:

description a pair (identifier, mode) describing a defined object;

access the set of descriptions of declared objects visible from the syntactic unit in question;

primode the mode (int or real) of a syntactic unit before applying coercion;

postmode the mode of a syntactic unit, determined by the outer context (coercion will yield this mode);

evaluable indicates whether the value of the syntactic unit can be computed statically;

value the value of the syntactic unit if it is computable statically;

id a unique representation of an identifier denotation.

The attribute sets are: $\begin{aligned} A I= & \{X . \text { access } \mid X \in\{\text { expression, primary, assignment, declaration }\}\} \\ & \vee\{X . \text { postmode } \mid X \in\{\text { expression, primary, assignment }\}\}\end{aligned}$

$A S=\{X$, primode $\mid X \in\{$ expression, primary, assignment $\}\}$ $\checkmark\{X$. evaluable $\mid X \in\{$ expression, primary $\}\}$

$\vee\{X$. value $X \in\{$ expression, primary, intconstant, realconstant $\}\}$

$\checkmark\{$ declaration description $\}$

$\checkmark\{$ identifier. id $\}$

The language is defined by:

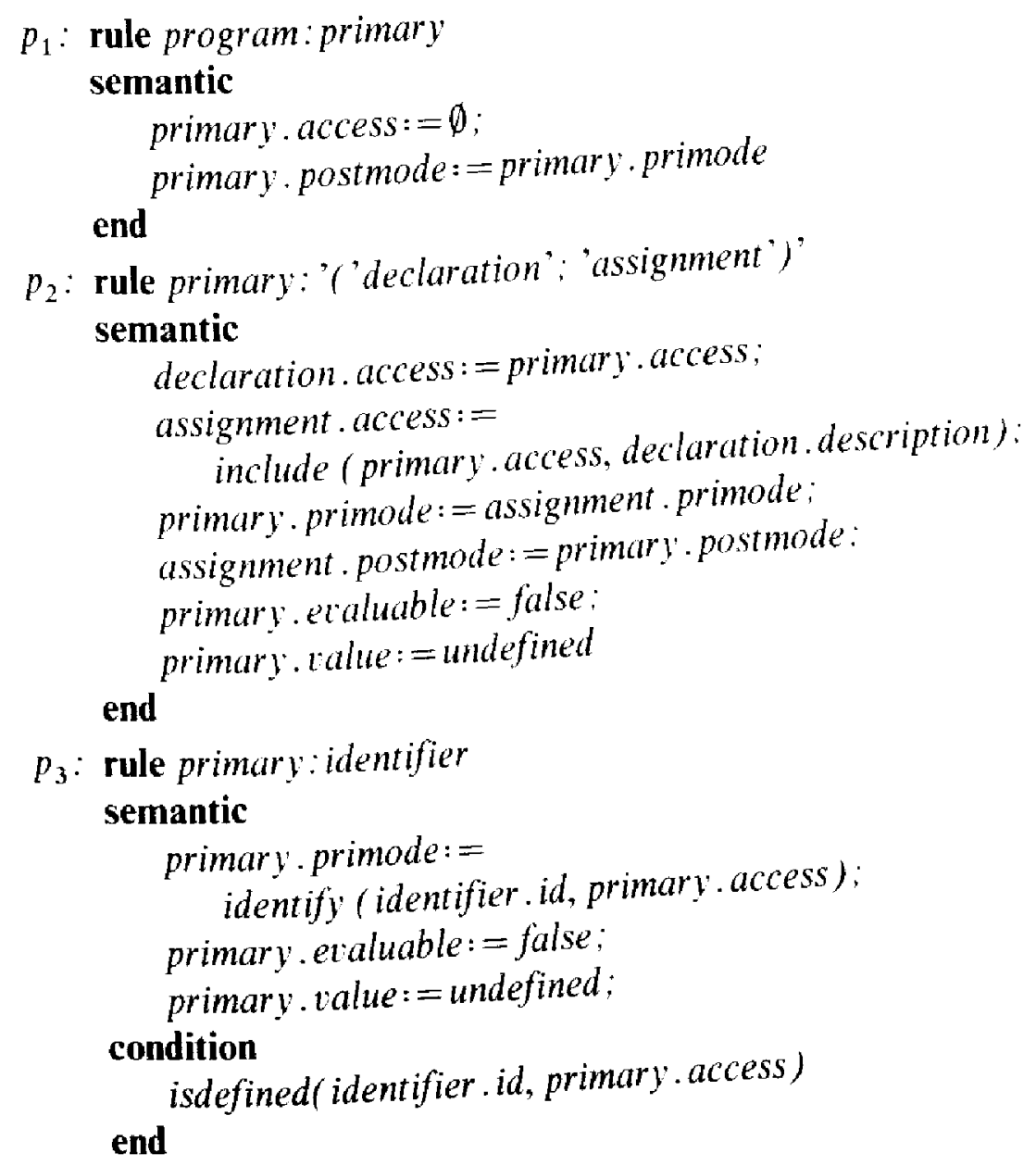


$p_{4}:$ rule primary : intconstant semantic

primary primode $:=i n t$;

primary evaluable $:=$ true;

primary value $:=$

if primary postmode = real

then widen(intconstant value)

end

else intconstant value fi

$p_{5}$ : rule primary:realconstant

semantic

primary primode $:=$ real;

primary evaluable $:=$ true

end

primary value $:=$ realconstant value

$p_{6}:$ rule assignment : identifier': = expression

semantic

expression. access $:=$ assignment .access;

assignment primode: $=$

identify (identifier.id, assignment access);

\section{condition}

expression .postmode: = assignment . primode;

isdefined(identifier.id, assignment.access) and

end

not (expression.primode $=$ real and

expression . postmode $=$ int )

$p_{7}:$ rule expression ${ }_{1}$ :expression $_{2}$ ' 'primary

semantic

expression $n_{2}$ access $:=$ expression $_{1}$. access;

primary access $:=$ expression $n_{1}$ access;

expression primode $=$

if expression, primode $=$ int

and primary primode $=i n t$

then int else real fi:

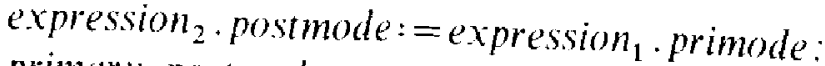

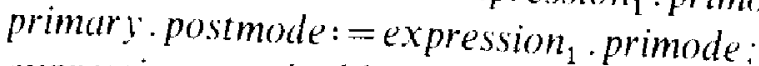

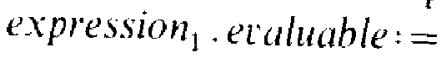

expression ${ }_{2}$ etaluable and primary. evaluable:

expression . $_{\text {calue }}:=$

if expression 1 evaluable

then add(expression 2 .value, primary.value)

end

else undefined fi

$p_{8}$ : rule expression: primary

semantic transfer

end 
$p_{9}$ : rule declaration: 'new' identifier' :='expression

\section{semantic}

expression access $:=$ declaration. access

declaration.description $:=$

(identifier.id, expression. primode);

expression postmode: =expression primode

end

The meaning of the functions include, isdefined, identify, add, and widen are given informally:

include $(a, d)$

if a set of descriptions, $d$ is a description; the result is $a \vee\{d\}$,
if $a$ does not contain a description $d^{\prime}$ for the same identifier described by $d,\left(a \backslash\left\{d^{\prime}\right\}\right) \vee\{d\}$ otherwise.

isdefined( $i d, a)$ a is a set of descriptions, id is an identifier; the result is true if $a$ contains a definition for $i d$, false otherwise.

identify ( $i d, a) \quad a$ is a set of descriptions, id is an identifier; the result is the mode defined for the identifier by a description in $a$. If no such description exists, the result is undefined.

$\operatorname{add}(v)$

widen $(v) \quad$ converts an integer value to a real value.
Rule $p_{2}$ describes the block structure of the language. For simplification only one

has the usual meaning for arithmetic values. declaration and one statement are allowed in each block. The scope rules described by the attribute access are defined as usual for block structured languages. The first semantic function of $p_{2}$ says that the declared identifier must not be applied in the declaration part. In order to enlarge the complexity of attribute dependencies the mode of a declared identifier is not given explicitly in rule $p_{9}$. It is determined by the mode of the initialization expression.

There are no semantic functions defining the attributes identifier.id, intconstant.value, and realconstant.value. Such attributes of terminal symbols are implicitly defined by the symbol text. Semantic conditions are headed by condition. transfer in $p_{8}$ is a shorthand notation for semantic functions which define corresponding attributes of expression and primary to have the same value

\section{Deciding Whether Attributed Grammars are Ordered}

In this section we introduce a method for deciding whether a given $A G$ is ordered. The method is based on the graph representation of dependency relations between attributes which was introduced by Knuth in [9]. The essential problem is to reduce a condition stated for the dependencies in the (infinite) set of sentences of a grammar to another condition stated for the dependencies between the finite set of attributes. The problem is solved by "projection" of the attribute dependencies in sentences into dependency relations associated to syntactic rules and symbols.

The basic idea of OAGs is the following: For each symbol $X \in V$ of a given $A G$ a partial order $D S_{X}$ over the attributes $A_{X}$ is constructed. ( $D S$ abbreviates dependencies between attributes of symbols.) It determines an evaluation order for 
the attributes, applicable in any context $X$ occurrs in. $(X \cdot a, X \cdot b) \in D S_{X}$ indicates that an attribute instance $K_{X} \cdot a$ must be evaluated before $K_{X} \cdot b$ (of the same $K_{X}$ ) for any symbol instance $K_{X}$ in any structure tree. If neither $(X \cdot a, X \cdot b)$ nor $(X \cdot b, X \cdot a)$ is in $D S_{X}$, the evaluation order of corresponding attribute instances can be chosen arbitrarily for any $K_{X}$.

The existence of such an order is a sufficient but not neccessary condition for the well-definedness of the AG (see Sect. 4). The relation $D S_{X}$ must comprise all direct and indirect dependencies, which may be derived from any possible context of $X$. Furthermore for different symbol occurrences in the same context the relations must be compatible with the dependencies between the attribute occurrences.

The evaluation order $D S_{X}$ is the base for the construction of a flexible and efficient attribute evaluation algorithm. It is closely adapted to the particular attribute dependencies of the AG. The principle is demonstrated here, for details see Sect. 5. Assume that an instance of $X$ is derived by

$$
S \Rightarrow u Y y \rightarrow_{p} u v X x y \rightarrow_{q} u v w x y \Rightarrow s .
$$

Then the corresponding part of the structure tree is

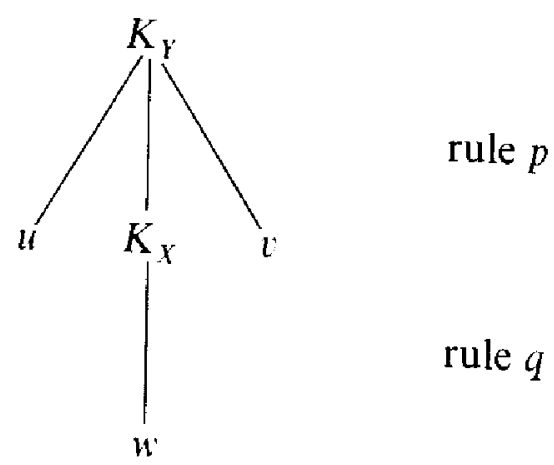

An attribute evaluation algorithm traverses the structure tree using the operations "move down to a descendant node" (e.g. from $K_{Y}$ to $K_{X}$ ) or "move up to the ancestor node" (e.g. from $K_{X}$ to $K_{Y}$ ). During a visit of a node $K_{Y}$ some attributes of $A F_{p}$ are evaluated according to semantic functions of $S F_{p}$, if $p$ is applied at $K_{Y}$. In general several visits to each node are needed until all attributes are evaluated. A local tree walk rule is associated to each $p$. It is a sequence of instructions of three types: move up to the ancestor, move down to a certain descendant, and
evaluate a certain attribute.

The relations $D S_{X}$ act as "interfaces" between visit-sequences, assuring that the visit-sequences for a tree node and for its descendants fit together in the following sense: A move down from $K_{Y}$ to $K_{X}$ is made in order to evaluate the attributes of a certain subset of $A S_{X}$. Their values can be used for further evaluations of functions in $S F_{p}$ after the traversal has returned to $K_{Y}$. The existence of such a relation $D S_{X}$ assures that the subset is the same for all rules $q$ deriving $X$. Correspondingly after a move to $K_{X}$ the subset of $A I_{X}$ of additionally evaluated Therefore each $D S_{X}$ for each occurrence of $X$ on the righthand side of any rule $p$. alternatingly inherited and syne a linear order over subsets of $A_{X}$, which contain ordered, since the evaluationthesized attributes only. In general $D S_{X}$ is partially interface. 


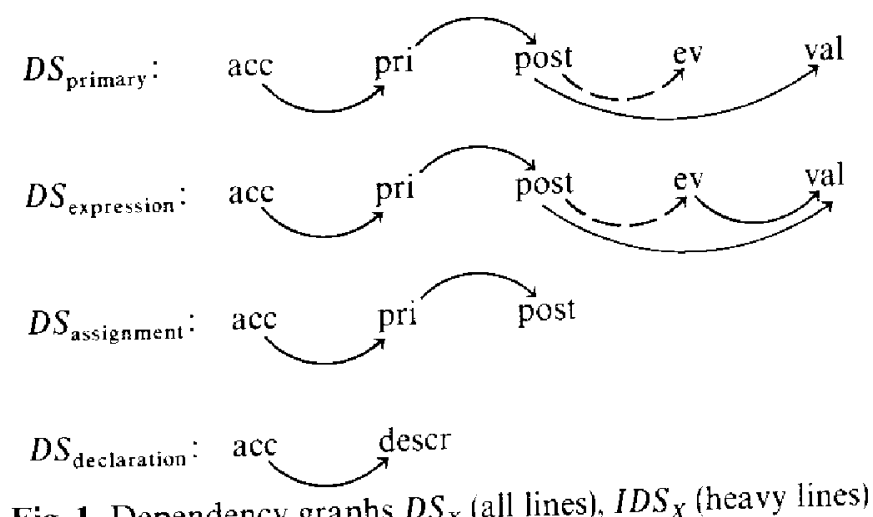

Fig. 1. Dependency graphs $D S_{X}$ (all lines). $I D S_{X}$ (heavy lines)

The $A G$ is an $O A G$, if a dependency relation $D S$ with the properties discussed above can be constructed according to the following definitions. Figure 1 shows the graph $D S$ for our example.

Definition 1.

$$
D P=\bigcup_{p \in P} D P_{p}, \quad D P_{p} \mathrm{c} A_{p} \times A_{p}
$$

is the relation of direct dependencies between attribute occurrences associated to production rules, where

$$
\begin{array}{r}
D P_{p}=\left\{\left(X_{i} \cdot a, X_{j} \cdot b\right) \mid \text { there is a semantic function in } S F_{p}\right. \\
\text { defining } \left.X_{j} \cdot b \text { depending on } X_{i} \cdot a\right\} .
\end{array}
$$

The relations $D P_{p}$ for our example are given in Fig. 2 by the heavy lines only. A dependency graph over the attribute instances in a structure tree for a sentence can be constructed by "pasting together" graphs $D P_{p}$ according to the applications of rules $p$ in the derivation of the sentence. Figure 3 shows such a graph for a sentence of our example.

In the next step we construct a dependency relation IDP over attribute occurrences. $I D P$ is defined recursively: Starting from $D P$ a direct or indirect dependency between attributes of one symbol occurrence induces a dependency between corresponding attributes of all occurrences of that symbol.

In the following we use the notation $D^{+}$for the non reflexive transitive closure of $D$. In the graph representation an $\operatorname{arc}(a, b)$ is in $D^{-}$iff an oriented path from $a$ to $b$ is in $D$.

\section{Definition 2.}

$$
I D P=\bigcup_{p \in P} I D P_{p}, \quad I D P_{p} \underline{\mathrm{c}} A_{p} \times A_{p}
$$

is the relation of induced dependencies between attribute occurrences, where

$$
\begin{array}{r}
I D P_{p}=D P_{p} \vee\left\{\left(X_{i} \cdot a, X_{i} \cdot b\right) \mid X_{i} \text { occurrs in rule } p, Y_{j} \text { occurrs in rule } q,\right. \\
\left.X_{i}=Y_{j}, 0 \leqq j \leqq n g,\left(Y_{j} \cdot a, Y_{j} \cdot b\right) \in I D P_{q}^{-}\right\} .
\end{array}
$$

The graphs $I D P_{p}$ for our example are shown in Fig. 2. 
$p_{1}$ :

program

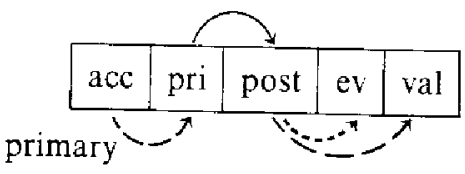

$\mathrm{p}_{3}$ :

primary

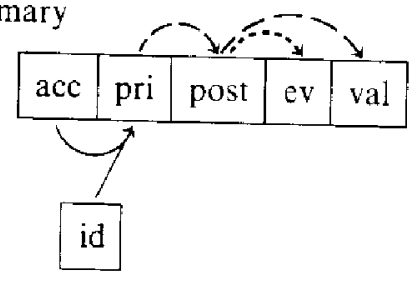

ident.

$p_{5}:$

primar

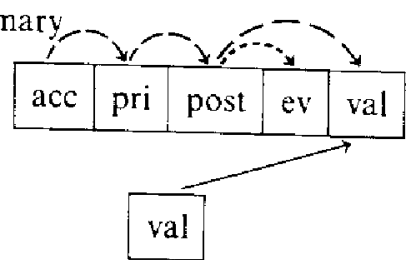

realconst

$p_{8}$ :

expr.

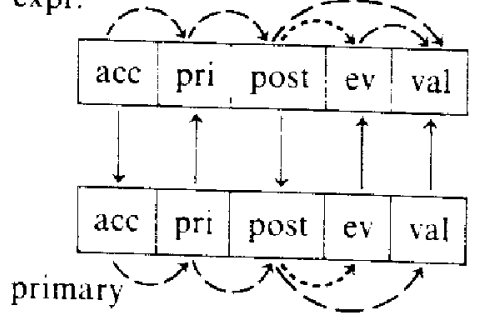

$p_{2}$ :

primary

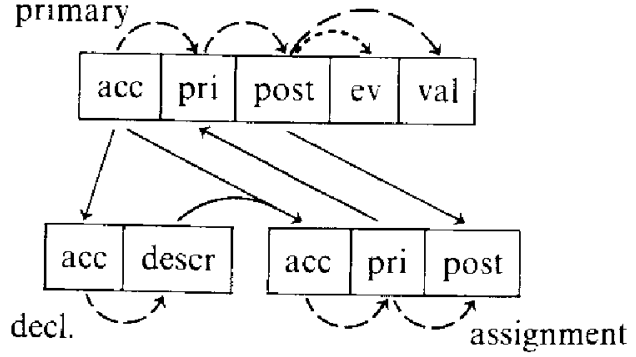

$p_{4}:$

primar

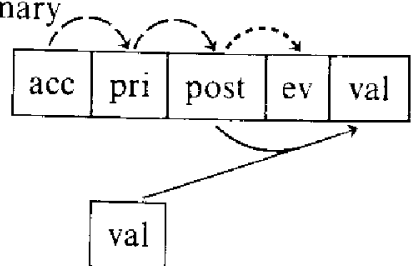

intconst

$p_{6}:$

assignment

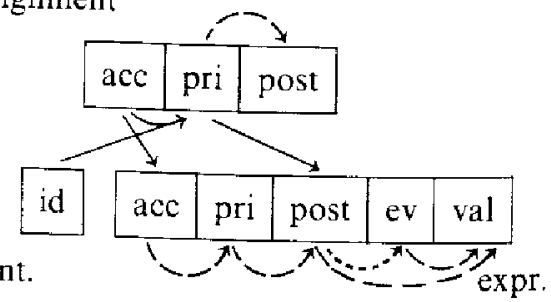

$p_{9}$ :

declaration

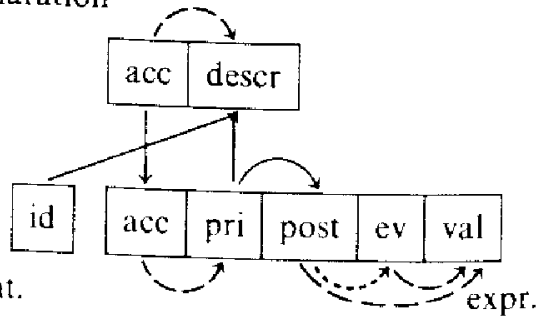
$p=$

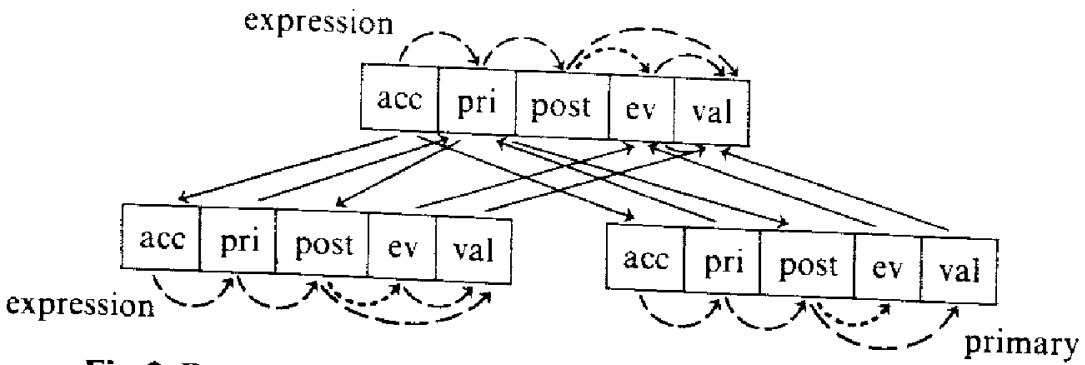

Fig. 2. Dependency graphs $D P_{p}$ (heavy lines), $I D P_{p}$ (heavy and dashed lines), $E D P_{p}$ (all lines) 


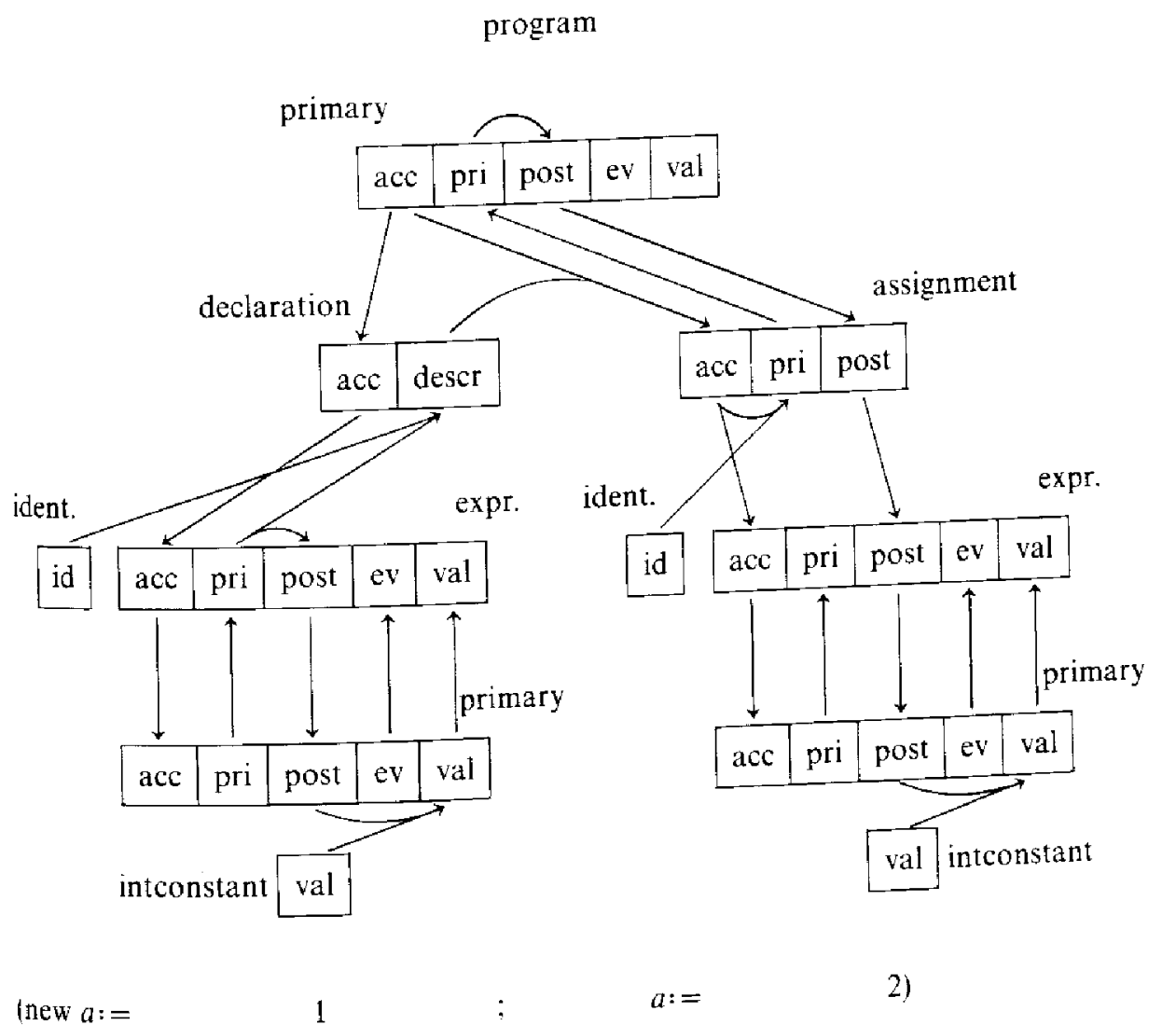

Inew $a$ : =

Fig. 3. Dependencies in an attributed structure tree

The construction of IDP "projects" indirect dependencies into dependencies between attribute occurrences. These dependencies are extracted in the next step. Definition 3.

$$
I D S=\bigcup_{X \in V} I D S_{X} \underline{\mathrm{c}} A \times A
$$

is the relation of induced dependencies between attributes of symbols, where

$I D S_{X}=\left\{(X \cdot a, X \cdot b) \mid\right.$ there is an $X_{i}=X$ in a rule $p$ and $\left.\left(X_{i} \cdot a . X_{i} \cdot b\right) \in I D P_{p}\right\}$.

Note. $(X \cdot a, X \cdot b) \in I D S$ implies that $\left(X_{i} \cdot a, X_{i} \cdot b\right) \in I D P_{p}$ for each occurrence $X_{i}$ of $X$ in each rule $p$. If there is a dependency between attribute instances $K_{X} \cdot a$ and $K_{X} \cdot b$ in the structure tree for any sentence of the language, $(X \cdot a, X \cdot b)$ is in IDS. On the other hand $(X \cdot a, X \cdot b) \in I D S$ does not imply that a dependency exists in any structure tree between corresponding attribute instances. In that sense IDS is "pessimistic". The graphs $I D S_{X}$ for our example are given in Fig. 2.

In the next steps $I D S$ will be completed to the relation $D S$ such that for each two attributes $X \cdot a \in A I, X \cdot b \in A S$ either $(X \cdot a, X \cdot b) \in D S$ or $(X \cdot b, X \cdot a) \in D S$ holds. Then each $D S_{X}$ defines a linear order over disjoint subsets $A_{X, k}$ of $A_{X}$. Each $A_{X, k}$ consists of those synthesized or inherited attributes whose values are additionally available after a move up or down in the tree. The evaluation order 


\begin{tabular}{l|ccccc}
$X=$ & $m_{X}$ & $A_{X .4}$ & $A_{X .3}$ & $A_{X .2}$ & $A_{X .1}$ \\
\hline primary & & & & & \\
expression & 4 & acc & pri & post & val, ev \\
assignment & 4 & acc & pri & post & - \\
declaration & 2 & & & acc & descr
\end{tabular}

Fig. 4. Disjoint partitions $A_{X, k}$

corresponds to decreasing order of the index $k$. Hence the subsets are defined such that $A_{X, k}$ contains attributes which contribute directly to the computation of attributes in $A_{X, k-1}$.

Definition 4. Let IDS be acyclic. For each $X \in V$ we define successively

$$
\begin{gathered}
A_{X, 1}=\left\{X \cdot a \in A S \mid \text { there is no } X \cdot b \text { such that }(X \cdot a, X \cdot b) \in I D S^{+}\right\}, \\
A_{X, 2 n}=\left\{X \cdot a \in A I \mid \text { for all } X \cdot b \in A_{X}:(X \cdot a, X \cdot b) \in I D S^{+}\right. \text {implies } \\
\left.X X \cdot b \in A_{X, m}, m \leqq 2 n\right\} \backslash \bigcup_{k=1}^{2 n-1} A_{X, k}, \\
A_{X, 2 n+1}=\left\{X \cdot a \in A S \mid \text { for all } X \cdot b \in A_{X}:(X \cdot a, X \cdot b) \in I D S^{+}\right. \text {implies } \\
\left.X \cdot b \in A_{X, m}, m \leqq 2 n+1\right\} \backslash \bigcup_{k=1}^{2 n} A_{X, k}
\end{gathered}
$$

until each $X \cdot a \in A_{X}$ is in an $A_{X . k}$. The sets $A_{X . k}$ form a disjoint partition of $A_{X}$ :

$$
\begin{aligned}
& A_{X}=\bigcup_{k=1}^{m_{X}} A_{X, k} \quad \text { for } m_{X} \geqq 1, \\
& A_{X, k} \wedge A_{X, j} \neq \emptyset \text { implies } k=j .
\end{aligned}
$$

Note. The disjoint partition could have been as well defined starting with the attributes evaluable first. In that case we had to decide whether the first subset contains inherited or synthesized attributes, i.e. whether attribute evaluation starts at the root or at the leafs of the tree. This assumption is not needed in Definition 4.

The disjoint partitions for our example are shown Fig. 4.

Definition 5 . Let $I D S$ be acyclic. The dependency relation $D S$ is defined as a completion of IDS:

$$
\begin{aligned}
& D S=\bigcup_{X \in V} D S_{X} \underline{\mathrm{c}} A \times A \\
& D S_{X}=I D S_{X} \vee\left\{(X \cdot a, X \cdot b) \mid X \cdot a \in A_{X, k}, X \cdot b \in A_{X, k-1}, 2 \leqq k \leqq m_{X}\right\} .
\end{aligned}
$$

In the last step we complete the dependency relation IDP according to the completion of $I D S$, in order to check that the completion does not cause cycles. Definition 6. The extended dependency relation over attribute occurrences EDP is defined as a completion of $I D P$ : 


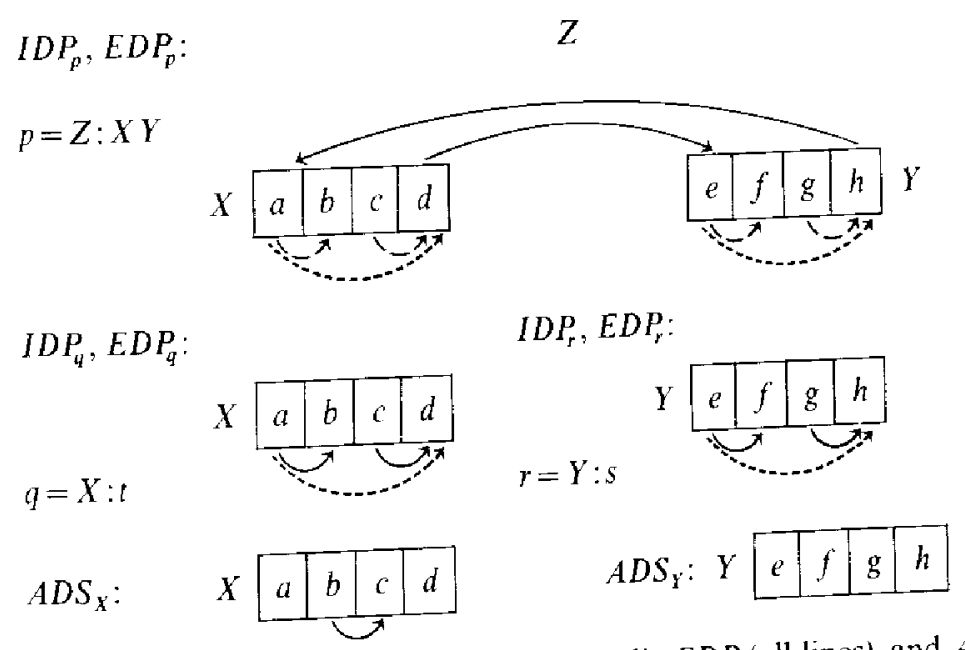

Fig. 5. Acyclic $I D P$ (heavy and dashed lines), cyclic EDP (all lines), and $A D S$

$$
\begin{aligned}
& E D P=\bigcup_{p \in P} E D P_{p} \\
& E D P_{p}=D P_{p} \vee\left\{\left(X_{i} \cdot a, X_{i} \cdot b\right)(X \cdot a, X \cdot b) \in D S, X_{i}=X\right\} .
\end{aligned}
$$

$D S$ is said to be compatible with the attribute dependencies if EDP is acyclic.

Definition 7. A given $A G$ is an $O A G$ iff the dependency relation $D S$ exists and is compatible with the attribute dependencies.

There exist AGs (usually not occurring in practical applications) where $D S$ is not compatible with the attribute dependencies. This situation is caused by the completion of the relations $I D S_{X}$. (Figure 5 gives an example.) If $I D P$ is acyclic one can show that each cycle in an $E D P_{p}$ contains at least two arcs, which are introduced by $\operatorname{arcs}(X \cdot a, X \cdot b),(Y \cdot c, Y \cdot d) \in D S \backslash I D S$, where $X$ and $Y$ correspond to different symbol occurrences in $p$. Furthermore $X \cdot a, X \cdot b$ and $Y \cdot c, Y \cdot d$ are independent in $I D S^{+}$. The arcs are in $D S$ because the attributes in question are associated to different subsets of $A_{X}$ and $A_{Y}$ according to Definition 4 .

If there are attributes independent in $I D S^{+}$one can enforce a different disjoint partition of $A$ by adding dependencies which do not correspond to semantic functions. An arbitrary set of dependencies $A D S \underline{\mathrm{c}} A \times A$ is called augmenting dependencies between attributes of symbols.

Definition 8. Let DP be the direct dependencies of an AG and ADS a set of augmenting dependencies. Let $D S^{\prime}$ be computed on the base of $D P^{\prime}$, where

$$
D P_{p}^{\prime}=D P_{p} \vee\left\{\left(X_{i} \cdot a, X_{i} \cdot b\right) \mid(X \cdot a, X \cdot b) \in A D S\right\} .
$$

An $A G$ is arranged orderly by $A D S$ if the $A G$ together with $D S^{\prime}$ is an $O A G$.

An AG is arranged orderly, if $A D S$ has the following property: It contains $\operatorname{arcs}(X \cdot b, X \cdot a)$ such that in each cycle of the original EDP at least one arc $\left(X_{i} \cdot a, X_{i} \cdot b\right)$ is replaced by $\left(X_{i} \cdot b, X_{i} \cdot a\right)$, and no new cycles are introduced. In general the computation of an $A D S$ with that property is a combinatorial problem of high complexity. An algorithmic solution shall not be discussed here because the problem has little practical relevance for AGs defining programming languages. For some restricted classes of AGs $A D S$ can be given easily (see next section). 


\section{Hierarchial Classification of Attributed Grammars}

In this section we compare OAGs with other classes of AGs with respect to the complexity of the expressible attribute dependencies. The expressive power of OAGs is larger than that of any class of AGs defined by a fixed evaluation strategy. Compared with well-defined AGs the restriction for OAGs does not exclude practical cases. The hierarchy of classes of AGs is listed below in descending order of expressive power:

AG attributed grammars

WAG well-defined attributed grammars [9]

ANCAG absolutely noncircular attributed grammars [8]

$\mathrm{OAG}$ ordered attributed grammars (defined in this paper)

$m$-APAG attributed grammars evaluable in $m$ alternating passes [5]

$n$-PAG attributed grammars evaluable in $n$ passes from left to right [1] $1-\mathrm{PAG}=\mathrm{L}-\mathrm{AG}$

attributed grammars evaluable in 1 pass from left to right [1], $L$ $L$-attributed grammars [13]

S-AG S-attributed grammars [13]

The definitions of the classes below the line are based on an apriori defined evaluation strategy, whereas for those above the line evaluation strategies must be computed from the attribute dependencies. In the following we shall discuss the relations between these classes. By simple examples it will be shown that the inclusions are strict.

The definition of absolutely noncircular AGs [8] can be derived from Definition 2 for OAGs:

An $A G$ is an ANCAG iff the following dependency relation is acyclic:

$$
\begin{aligned}
& I D P-A N C A G=\bigcup_{p \in P} I D P_{p}-A N C A G \\
& I D P_{p}-A N C A G=D P_{p} \vee \\
& \left\{\left(X_{i} \cdot a, X_{i} \cdot b\right) \mid i>0 \text { and there is a } q \in P:\right. \\
& \qquad \begin{aligned}
q & Y_{0}: w, \quad Y_{0}=X_{i}, \text { and } \\
& \left.\left(Y_{0} \cdot a, Y_{0} \cdot b\right) \in I D P_{q}-A N C A G^{+}\right\} .
\end{aligned}
\end{aligned}
$$

Obviously $I D P-A N C A G \subseteq I D P$, and for some AGs this inclusion is strict. Thus $O A G \underline{c} A N C A G$ is a strict inclusion. Figure 6 gives an example for an ANCAG which is not an OAG.

For each AG which is not well-defined there exists an attributed structure tree containing a cycle. Therefore IDP-ANCAG is cyclic and the AG is not absolutely noncircular. Figure 7 shows the dependencies of an AG, which is well-defined but not absolutely noncircular. Thus ANCAGc WAG is a strict inclusion.

Several classes of $\mathrm{AGs}$ are defined by apriori fixed strategies for attribute evaluation, such that for each sentence of $L(G)$ all attributes can be evaluated in 
Ordered Attributed Grammars

$$
\begin{aligned}
& D P_{p}: \quad S \quad D P_{q}: \quad S \\
& p=S: X t_{1}
\end{aligned}
$$

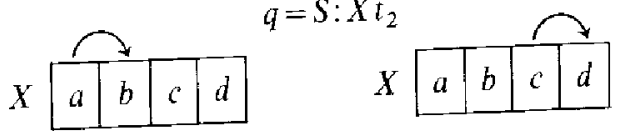

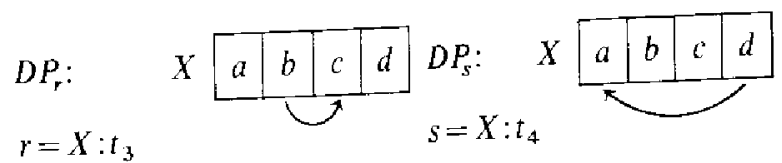

Fig. 6. $A N C A G$, but not $O A G$

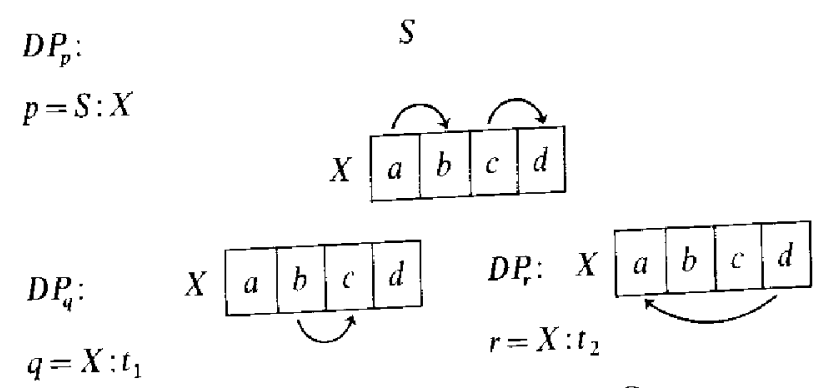

Fig. 7. WAG, but not ANCAG

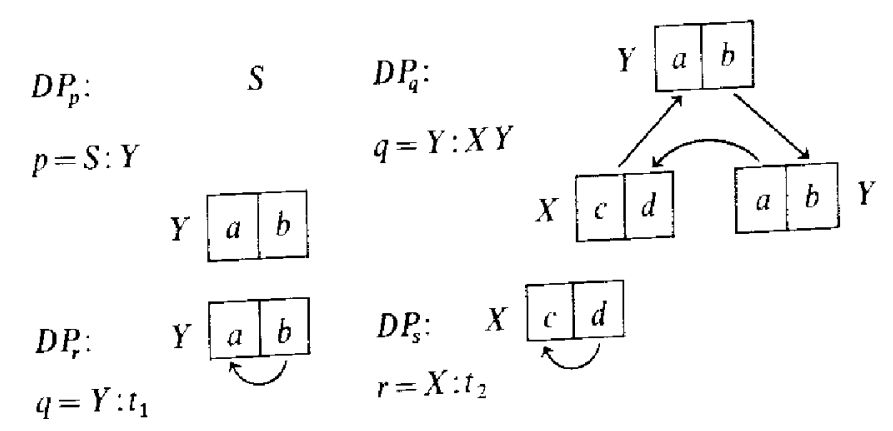

Fig. 8. 2-APAG, but not $n$-PAG

a certain number of passes over the structure tree. The most restrictive class contains the S-AGs defined in [13]. They only allow for synthesized attributes. which are evaluable in a single bottom-up pass.

Obviously they are included in 1-PAGs (called L-AGs in [13]), which are evaluable in a single top-down left to right pass. In [1] 1-PAGs are generalized to $n$-PAGs: For each $a \in A$ there is a number $1 \leqq k_{a} \leqq n$ such that any instance of $a$ in any structure tree is evaluated in the $k_{a}$-th top-down left to right pass. The number of passes needed is the maximum number $k_{a}$.

A further generalization leads to $m$-APAGs, which are evaluable in $m$ alternating top-down passes: the $i$-th pass proceeds from left to right (right to left) if $i$ is odd (even). For each $n$-PAG there is an $m \leqq 2 n-1$ such that it is an $m$-APAG, too. Figure 8 shows a situation for which the $m$-APAG condition holds but not the $n-P A G$ condition for any $n$.

An $m$-APAG is an OAG or can be turned into an OAG by augmenting dependencies. For any $m$-APAG $I D P$ and $I D S$ are acyclic. If the completion of $I D S$ leads to cyclic $E D P$, the $A G$ can always be arranged orderly:

Let $k_{a}$ be the number of the pass which evaluates the attribute $a$ according to the APAG strategy. Since the passes proceed top-down any instance $K_{X} \cdot a$ is 


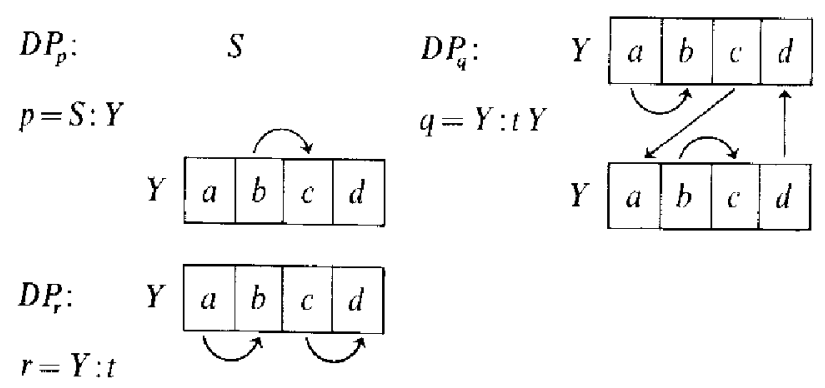

Fig. 9. OAG, but not $m$-APAG

evaluated before $K_{X} \cdot b$ if $k_{a}<k_{b}$ or $k_{a}=k_{b}$ and $a \in A I$ and $b \in A S$. Thus the $m$-APAG condition defines a dependency relation $I D S-A P A G \underline{\mathrm{c}} A \times A$ :

$$
I D S-A P A G=\left\{(X \cdot a, X \cdot b) \mid k_{a}<k_{b} \text { or }\left(k_{a}=k_{b}, a \in A I, b \in A S\right)\right\} .
$$

From Definition 3 follows that $I D S_{\mathrm{c}} I D S-A P A G$ for all $m-A P A G s$. Hence in the $O A G$ check neither $I D P$ nor $I D S$ can be cyclic. In a situation as shown in Fig. 5 the completion of $I D S$ causes $E D P$ to be cyclic. In any such case the $A G$ can be arranged orderly by $A D S=I D S-A P A G$. So the class of $m$-APAGs is included in the class of OAGs in the sense that each $m$-APAG can be arranged orderly.

The inclusion is strict because there are OAGs which can not be evaluated in $m$ alternating passes for any $m$. Such AGs contain recursive syntactic rules with attribute dependencies such that the number of evaluation passes is determined by the unlimited recursion depth (as shown in Fig. 9).

\section{Visit-Sequences for Ordered Attributed Grammars}

OAGs (and the larger classes as well) do not imply a predefined strategy for the walk through the structure tree during attribute evaluation. For each grammar of this class a special evaluation algorithm can be constructed based on the attribute dependencies. Such an algorithm implements the semantic analysis of a compiler for the defined language. It is independent of the compilation of any particular sentence: therefore it can be constructed at time of compiler generation. The situation is similar to the construction of a parsing algorithm from syntactic definitions.

The construction is based on the following idea: For each rule $p \in P$ a visitsequence $V S_{p}$ is computed. A visit-sequence is a local tree walk rule applied at each node of the structure tree, which is derived by $p$. It describes the order of visits to surrounding nodes and of evaluations of semantic functions between those visits. (It is assumed that the semantic functions are translated conveniently into the implementation language of the compiler. Only the applicability of the functions according to the attribute dependencies is considered here.)

The ordering property of an attributed grammar yields both a rather simple construction of the visit sequences, and a rather simple implementation of the attribute evaluation algorithm. The visit-sequences for OAGs are linear sequences of actions (node visits and attribute evaluations). The next action to be executed 
at evaluation time is completely determined by its predecessor. The comparable evaluation rules for ANCAGs ([8] and [3]) are partially ordered graphs. The next executable action is determined by its predecessor and a context dependent set of attributes already evaluated. Thus the evaluation algorithm is simpler for OAGs than for ANCAGs (see Sect. 6).

The construction of the visit-sequences is based on the dependency relation EDP defined in Sect. 3. As attribute evaluation may be done interleaved with the construction of the structure tree, we shall distinguish three cases for the construction of the visit-sequences:

TC: Attribute evaluation starts, when the whole structure tree is completed. BU: Attribute evaluation is done interleaved with bottom-up tree construction. TD: Attribute evaluation is done interleaved with top-down tree construction.

For each rule $q$ a visit-sequence $V S_{q}$ will be constructed separately. For any pair of rules $q=Y: u X v$ and $r=X: w V S_{q}$ and $V S_{r}$ are constructed such that they fit together in the sequence of attribute evaluation and the moves between context $q$ and context $r$ (ancestor and descendant visits). This interface is defined by the disjoint partition of $A_{X}$ (Definition 4). Such an interface for the TC-and TD-case and $m_{X}=6$ is given below:

$$
\begin{aligned}
& \text { context } q
\end{aligned}
$$

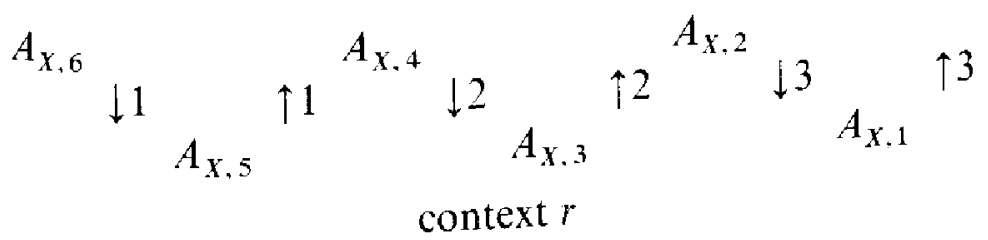

The inherited attributes $\left(A_{X . m}\right.$ for even $\left.m\right)$ are evaluated in the context $q$, the synthesized $\left(A_{X . m}\right.$ for odd $m$ ) in the context $p . \downarrow k$ denotes the $k$-th descendant visit from $q, k$ denotes the $k$-th ancestor visit from $r$. The number of ancestor and descendant visits are both

$$
n v_{X}=f_{X} \operatorname{div} 2,
$$

where $f_{X}$ is the smallest even number $f_{X} \geqq m_{X}$ (for the TD-case).

In the BU-case tree construction and attribute evaluation starts from the leafs of the tree. Thus the first visit in the interface moves in upward direction:

context $q$

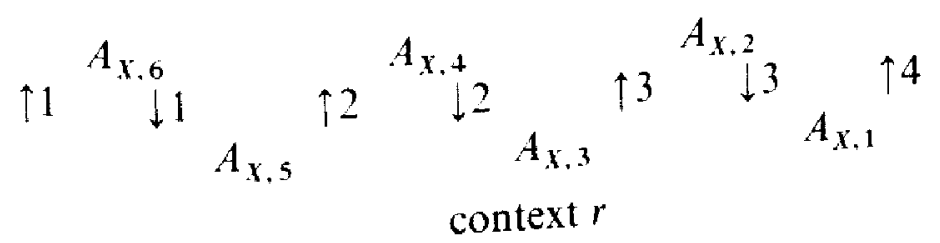

In the BU-case the number of ancestor and descendant visits is

$$
n v u p_{X}=\left(f_{X}+1\right) \operatorname{div} 2, \quad n v d o w n_{X}=n v u p_{X}-1
$$

where $f_{X}$ is the smallest odd number $f_{X} \geqq m_{X}$. 
The construction of $V S_{p}$ replaces in the graph $E D P_{p}$ all attribute occurrences not evaluated in the context $p$ by corresponding ancestor or descendant visits.

Definition 9. Each visit-sequence $V S_{p}$ associated to a rule $p \in P$ is a linearly ordered relation over defining attribute occurrences and visits:

$$
\begin{aligned}
& V S_{p} \underline{\mathrm{c}} A V_{p} \times A V_{p}, \quad A V_{p}=A F_{p} \vee V_{p}, \\
& V_{p}=\left\{v_{k, i} \mid 0 \leqq i \leqq n p, 1 \leqq k \leqq n v_{X}, X=X_{i}\right\} .
\end{aligned}
$$

$v_{k, 0}$ denotes the $k$-th ancestor visit, $v_{k, i}, i>0$ denotes the $k$-th visit of the descendant $X_{i}$. The function MAPVS maps nodes of EDP to nodes of VS:

$$
\begin{aligned}
& \operatorname{MAPVS}\left(X_{i} \cdot a\right):= \begin{cases}X_{i} \cdot a & \text { if } X_{i} \cdot a \in A F_{p} \\
\varepsilon_{k, i} & \text { if } X_{i}=X, \quad X_{i} \cdot a \in\left(A_{X, m} \wedge A C_{p}\right), \\
& k=\left(f_{X}-m+1\right) \operatorname{div} 2, k>0 \\
\text { undefined } & \text { if } X_{i}=X, \quad X_{i} \cdot a \in\left(A_{X, m} \wedge A C_{p}\right), \\
0 & =\left(f_{X}-m+1\right) \operatorname{div} 2 .\end{cases} \\
& {\left[S_{p}=\left\{\left(M A P V S\left(X_{i} \cdot a\right), M A P V S\left(X_{j} \cdot b\right)\right) \mid\left(X_{i} \cdot a, X_{j} \cdot b\right) \in E D P_{p}\right\} \vee\right.} \\
& \text { \{arbitrary arcs such that } V S_{p} \text { is linearly ordered and } \\
& \left.c_{k, 0}, k=n v_{X}, X=X_{0} \text { is the "largest" element }\right\} .
\end{aligned}
$$

Note that attributes evaluated before the context $p$ is entered the first time (the "smallest" inherited attributes of $X_{0}$ in the TC-case and the "smallest" synthesized attributes of all $X_{i}, i>0$ in the BU-case) are not represented by a visit. Usually all attributes of terminal symbols are synthesized and implicitly defined by the symbol text. Thus terminal nodes need not be visited.

The TD-case is similar to the TC-case. Additionally for each $V S_{p}$ it must be assured that the first visits of the descendants are ordered from left to right:

(a) $0<i<j \leqq n p$ implies $\left(v_{1, i}, v_{1, j}\right) \in V S_{p}^{+}$,

(b) $0<i \leqq n p$ implies $\left(c_{1, i}, v_{1,0}\right) \in V S_{p}^{+}$.

The visit-sequences are computed as described for the TC-case. The freedom of the arbitrary linearisation is used in order to achieve the above condition. In general that is not possible for all $V S_{p}$. Thus we consider all symbols which would be visited "too late" for the first time in some context $p$ :

$$
\begin{gathered}
L . A T E=\left\{X \mid \text { there is a } p \text { such that } X=X_{i}, i>0, \text { and } v_{1, i}\right. \text { occurrs } \\
\text { "too late" in } \left.S_{p} \text { according to (a) or (b) }\right\} .
\end{gathered}
$$

The set $L A T E$ contains all symbols $X$, for which the interface, i.e. the disjoint partitions of the attributes, must be changed such that the visit-sequences $X$ occurrs in fit to conditions (a) and (b). Thus we add an empty set $A_{X, k}, k=m_{X}+1$. to the disjoint partitions of each $X \in L A T E$, if $m_{X}$ is even. (If $m_{X}$ is odd, the first visit of $X$ does not depend on attributes and can be placed at an appropriate position in any visit-sequence.) Then the visit-sequences are recomputed using the updated interfaces. The added sets $A_{X, k}$ will yield an additional visit of $X$ for syntactic purpose only. It can be placed such that (a) and (b) hold, because it does not depend on any attributes. 
In general some recomputed visit-sequences $V S_{q}$, for $q=Y: w$ and $Y \in L A T E$ violate condition (b), because the interface for $Y$ changed. Therefore we iterate the computation of a new set $L A T E$ and new visit-sequences until the conditions bold for all $V S_{q}$. The iteration terminates because the sets $L A T E$ are disjoint for all iteration steps, and $V$ is finite.

The evaluation of the semantic condition $S C_{p}$ can be inserted in $V S_{p}$ at any place after the evaluation of the attributes it depends on.

The visit-sequences for our example (for the TC-case) can be computed as follows:

$V S_{1}=$ primary access, $v_{1, \text { primary }}$, primary postmode, $v_{2, \text { primary }}, v_{1, \text { ancestor }}$

$V S_{2}=$ declaration .access, $v_{1, \text { declaration }}$, assignment access, $v_{1, \text { assignment }}$, primary primode, $v_{1, \text { ancestor }}$, primary evaluable, primary value, assignment postmode, $v_{2, \text { assignment }}, v_{2, \text { ancestor }}$

$\mathrm{VS}_{3}=$ condition, primary . primode, $v_{1 \text {, ancestor }}$, primary. evaluable, primary. value,

$v_{2, \text { ancestor }}$

$V S_{4}=V S_{5}=$

primary primode, $v_{1, \text { ancestor }}$, primary evaluable, primary. value, $v_{2 \text {,ancestor }}$

$V S_{6}=$ expression access, $v_{1, \text { expression }}$, assignment . primode, $v_{1, \text { ancestor }}$.

expression . postmode, condition, $v_{2, \text { expression }}, v_{2, \text { ancestor }}$

$V S_{7}=$ expression 2 . access, $v_{1 \text { expression }}$, primary access, $v_{1, \text { primary }}$,

expression 1 . primode, expression 2 . postmode, $v_{2, \text { expression }}$,

primary postmode, $v_{2, \text { primary }}, v_{1, \text { ancestor }}$,expression ${ }_{1}$.evaluable,

expression 1 . value, $v_{2, \text { ancestor }}$

$V S_{8}=$ primary. access, $v_{1, p r i m a r y}$, expression.primode, $v_{1, \text { ancestor }}$, primary.postmode,

$\mathrm{IS}_{9}=$ expression. access, $v_{1, \text { expression }}$, expression postmode, $v_{2, \text { expression }}$.

declaration.descr, $v_{1, \text { ancestor }}$

\section{Implementation of Visit-Sequences}

The compiler phase for semantic analysis traverses through the structure tree and computes the attribute values. The control flow of this algorithm is exactly given by the visit-sequences. They describe which tree nodes are to be visited and which semantic functions are to be called. The visit-sequences can be translated into recursive procedures or coroutines or into the transition table of an automaton. The semantic functions (translated into the implementation language of the compiler) complete the compiler phase. (The situation is comparable to LLgrammars, which can be parsed by recursive procedures or table driven parsers, both augmented by actions appended to production rules.) In general a table driven algorithm is the most efficient implementation. An implementation using recursive procedures or coroutines is well suited to extend a recursive descent parsing algorithm.

In this section we show the principles of four implementation techniques. We assume that the visit-sequences are constructed such that attribute evaluation starts when tree construction is completed. It will be obvious how the implemen- 
tations must be modified if the attributes are evaluated interleaved with tree construction.

\subsection{Implementation Using Coroutines}

The implementation technique given here uses SIMULA-classes as coroutines It can be easily transferred to other programming languages providing comparable control structures. We assume that each node of the structure tree is represented by an instance of a class. The type of the class is determined by the corresponding syntactic rule $p$. The tree structure is represented by references from a node to its descendants.

For each rule $p=X_{0}: X_{1} \ldots X_{n}$ a class definition is constructed. For rule $p_{2}$ of our example it reads as follows:

node class $p-2$;

begin ref (node) declaration, assignment

attributes access, primode, postmode, evaluable, value;

(* syntactic part:

the subtress for declaration and assignment are constructed *) detach ;

(* semantic part: *)

declaration.access;

call (declaration);

assignment access: $=\ldots$;

call (assignment).

primode $:=\ldots$;

detach:

eraluable: $=\ldots$;

valie: $=\ldots$;

assignment . postmode: $=\ldots$;

call (assignment):

end

detach

The semantic part is a straightforward implementation of the visit-sequence $1 S_{p}$ : An ancestor visit is translated into a detach-statement. A visit of descendant $X_{i}$ is translated into a coroutine call call $\left(X_{i}\right)$. Each attribute $X_{i} \cdot a$ in the visit-sequence is translated into a call of a semantic function in $S F_{p}$ defining its value.

\subsection{Implementation $U$ sing Recursice Procedures}

We assume that the nodes of the structure tree are represented by data structures with components for the attributes, references to the descendants, and a component indicating the derivation rule applied to the node. For each rule $p=X_{0}$ : $X_{1} \ldots X_{n}$ the visit-sequence $V S_{p}$ is split into $m$ parts each terminated by an ancestor
visit:

$$
V S_{p}=V S_{p, 1}, V S_{p, 2} \ldots V S_{p, m}
$$


For each $V S_{p, i}$ a procedure $p^{i}$ is constructed. For rule $p_{2}$ of our example it reads as follows:

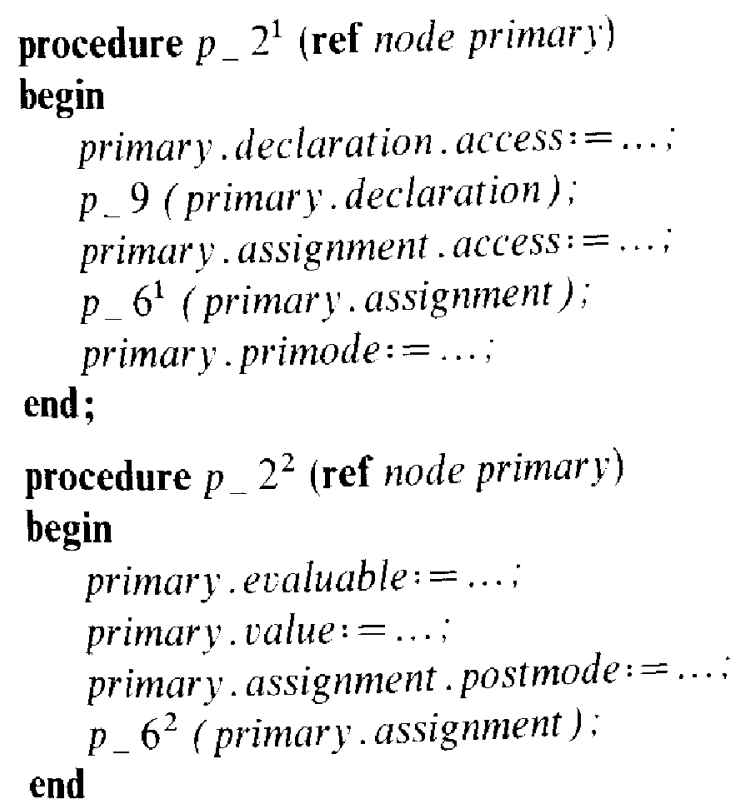

The body of the procedure is a straightforward translation of the $i$-th part of the visit sequence $V S_{p, i}$ : Each $X_{i} \cdot a$ is translated into an appropriate call of a semantic function, and each descendant visit $v_{k, i}$ into a call $q^{k}\left(X_{i}\right)$. If there is more than one rule $q$ for the $i$-th descendant $X_{i}$ the $r$-th visit $r_{r . i}$ is translated into a case statement, which determines the procedure to be called by inspecting the rule_indicator of $X_{i}$ :

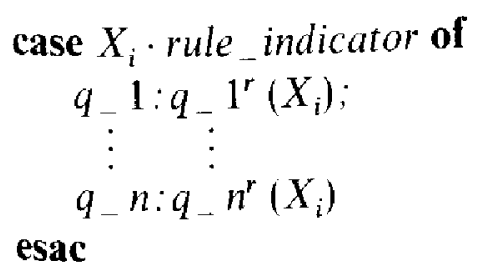

The ancestor visit at the end of $V S_{p . i}$ is implicitly implemented by the return from the procedure at the end of the body.

\subsection{Implementation by a Stack Automaton}

This implementation technique can easily be deduced from the previous one using recursive procedures: The structure tree is represented as in 6.2. All parts of all visit-sequences $V S_{p, r}$ as defined above are collected into a transition table. It is comparable to the program text of the procedure bodies in 6.2 . A stack is maintained containing pairs of a reference to a treenode and the next position in the table to be encountered. It is an explicit implementation of the runtime stack needed for the procedures of 6.2 , which contains a return address and the node reference for each procedure incarnation. An additional function $M A P$. $D O W N$ is provided mapping the visit number $r$ of a descendant visit $v_{r, i}$ and the rule indicator $p$ of the visited node into the first table entry of $V S_{p . r}$ (comparable to the case statements of 6.2). The control loop of the automaton then has the following structure: 


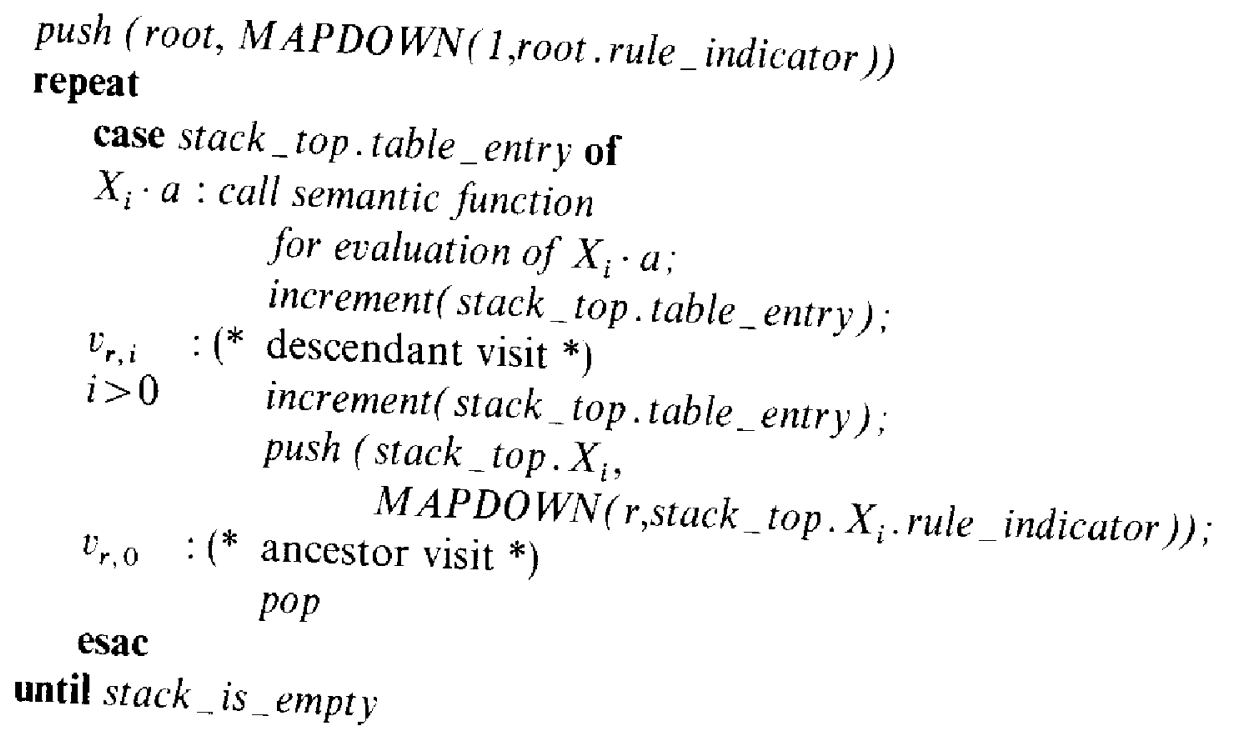

\subsection{Implementation by a Finite Automaton}

This implementation technique avoids the (implicit or explicit) stack of the previously described techniques. We assume that the tree is linked both upwards number $s$, when the node is the $s-$ ains an indicator of the applied rule and the $M A P D O W N$ and MAPUP the $s$-th descendant of its ancestor. Two functions of the currently visited node and the rule indistor visit, the descendant number $s$ element of $V S_{p}$, which is preceeded by indicator $p$ of its ancestor into that then has the following structure:

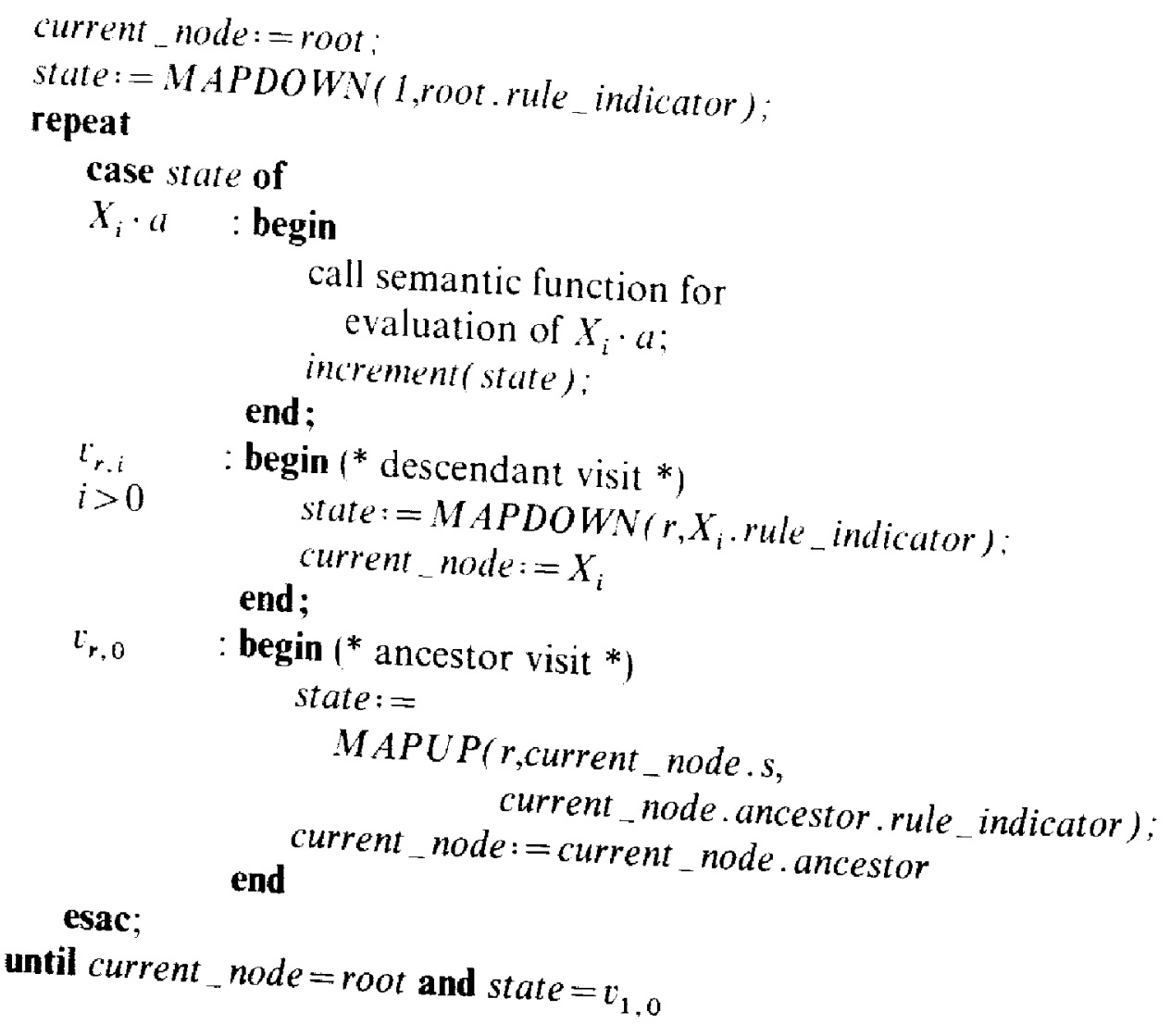




\section{An Algorithm for Computing Visit-Sequences}

This section discusses an abstract algorithm which checks whether a given AG is ordered, and computes the visit sequences for it. We show that in the worst case the complexity of the algorithm is bound by the product of the $4^{\text {th }}$ power of the maximum number of attributes associated to a symbol, the $3^{\text {rd }}$ power of the maximum length of a syntactic rule, and the length of the underlying context-free grammar. This is an important result compared to the intrinsic exponential complexity of well-definedness of AGs [4]. For most programming languages the number of attributes is rather small. Thus the complexity of the algorithm is suitable small for practical applications. The complexity of the algorithm is expressed by the size of the input grammar. The parameters used are:

$|P|$ the number of syntactic rules;

$|R|$ the maximum number of symbols in a single syntactic rule;

$|X|$ the maximum number of attributes of a single symbol;

$|V|$ the number of symbols, bound by $|V| \leqq|G|$.

These parameters are used to compute the following expressions:

$|G|=|P||R|$ the length of the grammar;

$|D|=|R||X|$ the maximum number of nodes of a dependency graph for a rule;

We shall describe the algorithm in form of a program for an abstract machine, which is defined by the following data structures and functions.

A dependency relation $D$ over $n$ attributes is considered as a graph with $n$ nodes. The arcs $(a, b) \in D$ are represented by a vector of $n$ sets over $n$ nodes. $D$ is initially empty. Primitive operations on $D$ are $(a, b) \in D,(a, b) \notin D$ and addarc $(D,(a, b))$ which adds the arc $(a, b)$ to $D$. The costs of these operations are $O(1)$.

Each rule $p=X_{0}: X_{1} \ldots X_{n p}$ is represented by a vector $X$ of symbol occurrences, a dependency relations $T D P_{p}$ over the attribute occurrences $X_{i} \cdot a$, and a graph $V S_{p}$ representing the visit-sequence.

For each symbol $X \in V$ there is a linked list of its occurrences, and a dependency graph $T D S_{X}$ over the attributes $A_{X}$. The arcs of this graph are marked if they are already induced at all occurrences of $X$. A vector $P A R T$ maps the attributes $X \cdot a$ to the index $k$ of $A_{X, k}$.

The following functions are used for updating dependency relations.

addarctrans $(D,(a, b))$ :

It is assumed that $D$ is a transitively closed, non-reflexive relation. The arc $(a, b)$ is added to $D$. All arcs needed to close $D$ transitively again are added to $D$, too. The costs of this operation are $\mathrm{O}\left(n^{2}\right)$, if $D$ is a graph over $n$ nodes. addarcinduce $(D,(a, b))$ :

This function is applied to relations $T D P_{p}$ associated to rules $p$. It updates the transitive closure $D$ as addarctrans does. Additionally, for each new arc $\left(X_{i} \cdot a, X_{i} \cdot b\right)$ an $\operatorname{arc}(X \cdot a, X \cdot b)$, which is not marked, is added to $T D S_{X}$, if $(X \cdot a, X \cdot b) \notin T D S_{X}, X=X_{i}$. The costs of this function are the same as the costs of addarctrans, because the additional action is only applied to a subset of the totally inspected arcs.

The main idea of the algorithm is the following: Instead of the dependency relations $D P_{p}, I D P_{p}, E D P_{p}$. their transitive closures are computed. The recursive 
definitions of Sect. 3 are transformed into iterative algorithms. The algorithm consists of 5 steps: Steps 1 and 2 compute the relations $I D P_{p}^{+}$and $D S_{X}^{+}$. Step 3 computes the disjoint partitions. In step $4 E D P^{+}$is computed and it is decided whether the AG is ordered. Step 5 computes the visit-sequences.

Step 1. Computation of $D P^{+}$

Input: The semantic functions $S F$.

Output: Dependency graphs $T D P_{p}=D P_{p}^{+}$, and updated graphs $T D S_{X}$.

Method: For each dependency established by a semantic function $f \in S F_{p}$ an arc is added to the transitively closed $T D P_{p}$ by calling addarcinduce.

for each $f \in S F_{p}$ defining $X_{j} \cdot b$

loop for each argument $X_{i} \cdot a$ of $f$

loop if $\left(X_{i} \cdot a, X_{j} \cdot b\right) \notin T D P_{p}$ repeat

then addarcinduce $\left(T D P_{p},\left(X_{i} \cdot a, X_{j} \cdot b\right)\right)$ fi

repeat The graphs $T D S_{X}$ contain the arcs to be induced due to direct dependencies; they
are not marked.

Complexity: $\mathrm{O}\left(|P||D|^{4}\right)$

The upper bound for the number of arcs in $T D P_{p}$ is $|D|^{2}$; it is multiplied with $|D|^{2}$, the complexity of addarcinduce.

Step 2. Computation of $I D P$

Input: Acyclic dependency graphs $T D P_{p}=D P_{p}^{+}$, graphs $T D S_{X}$ containing arcs to be induced due to direct dependencies.

Output: Dependency graphs $T D P_{p}=I D P_{p}^{+}$containing all induced arcs according to Definition 2. and the graphs $T D S_{X}=I D S_{X}^{+}$.

Method: Each arc in TDS which is not marked is induced at each occurrence of the symbol. If new arcs to be induced are found they are added to TDS by addarcinduce.

while there is an $\operatorname{arc}(X \cdot a, X \cdot b)$ in $I D S$ which is not marked

loop mark $(X \cdot a, X \cdot b)$;

for each occurrence $X_{i}$ of $X$ in any rule $p$

loop if $\left(X_{i} \cdot a, X_{i} \cdot b\right) \notin T D P_{p}$

then addarcinduce $\left(T D P_{p},\left(X_{i} \cdot a, X_{i} \cdot b\right)\right)$

fi

repeat

repeat

The invariant condition for the while-loop is: If a $T D P_{p}$ contains an $\operatorname{arc}\left(X_{i} \cdot a\right.$, $\left.X_{i} \cdot b\right)$, and if $(X \cdot a, X \cdot b)$ is marked, then $(X \cdot a, X \cdot b)$ is already induced in all $I D S_{X}^{+}$.

Complexity: $\mathrm{O}\left(|G||X|^{2}|D|^{2}\right)$ 
There are at most $|X|^{2}$ arcs in each $T D S_{X}$. The for-loop is executed once for each arc and each occurrence of the symbol. There are $|G|$ symbol occurrences where arcs may be induced. The marking of arcs in TDS ensures that no arc is induced more than once.

Step 3. Computation of the disjoint partitions of $A_{X}$

Input: Acyclic dependency graph $T D S=I D S^{+}$.

Output: A vector PART mapping $a \in A_{X}$ to $k$ if $X \cdot a \in A_{X, k}$ according to Definition 4.

Method: Starting with $k=1$ for each attribute it is checked, whether it can be included into $A_{X, k}$. When no more attributes can be added to $A_{X, k}, k$ is incremented.

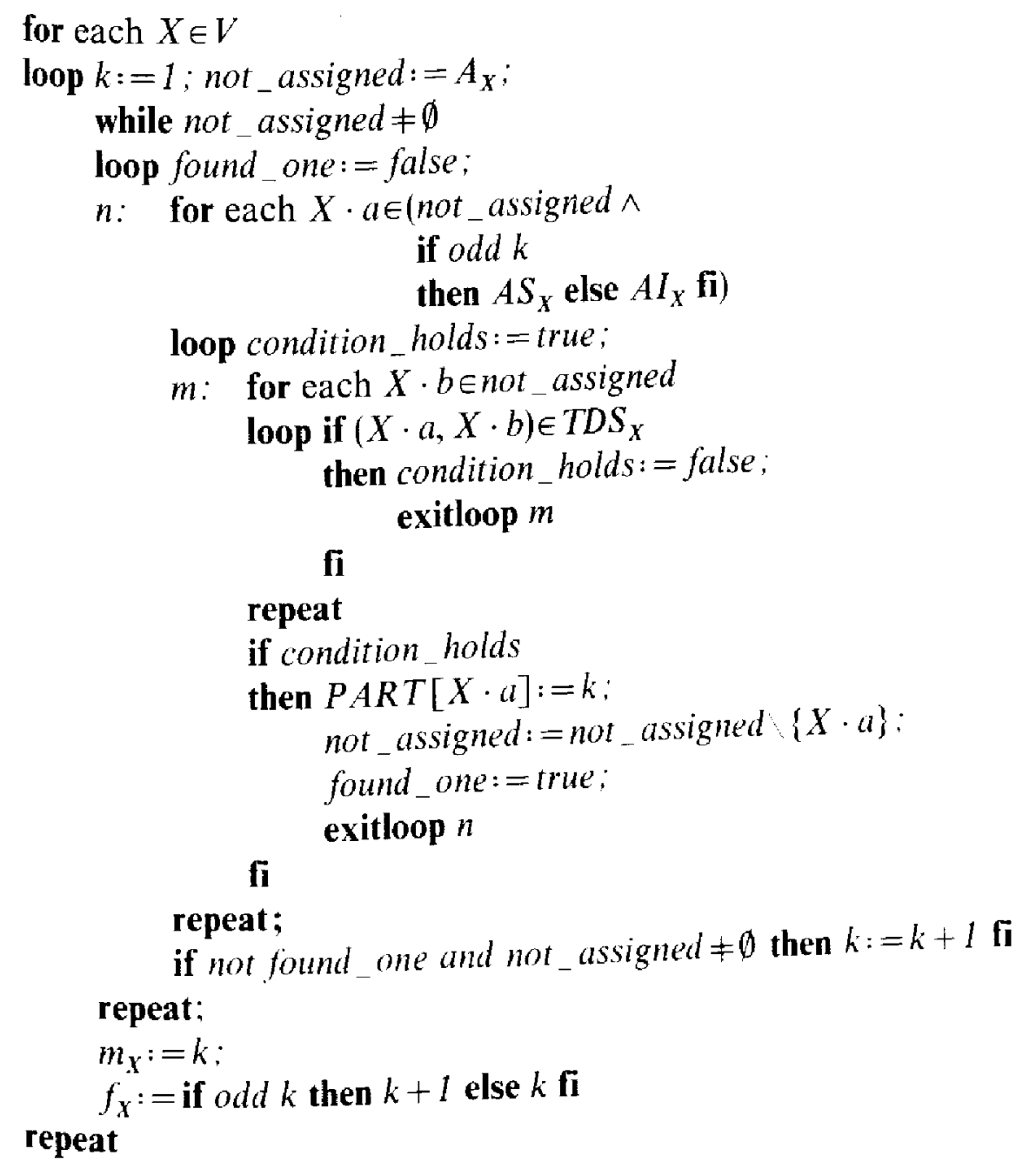

\section{Complexity: $\mathrm{O}\left(|V||X|^{3}\right)$}

In the worst case each $A_{X . k}, k>1$ contains one attribute, and $A_{X .1}$ is empty. Thus the while-loop is repeated at most $2|X|+1$ times. $(|X|$ steps succeed in assigning $X \cdot a$ to a subset, and $|X|+1$ steps fail.) In the loop $n$ success or failure is determined after at most $|X|$ steps. The loop $n$ checks the condition for not more than $|X|$ arcs. 
Step 4. Computation of EDP

Input: Graphs $T D P_{p}$ and the vector $P A R T$ describing the disjoint partitions of the $A_{X}$.

Output: Graphs $T D P_{p}=E D P_{p}^{+}$

Method: Arcs are added to TDP according to the relation given by $P A R T$.

for each $p \in P$

loop for $i:=0$ to $n p$

loop $X:=X_{i}$;

for each $X \cdot a$

loop for each $X \cdot b$

loop if $P A R T[X \cdot a]>P A R T[X \cdot b]$

then addarctrans $\left(T D P_{p},\left(X_{i} \cdot a, X_{i} \cdot b\right)\right)$

fi

repeat

repeat

repeat

repeat

Now $T D P_{p}=E D P_{p}^{+}$holds for each $p \in P$. If each $T D P_{p}$ is acyclic the $A G$ is an $O A G$ Complexity: $\mathrm{O}\left(|P||R||X|^{2}|D|^{2}\right)$

The complexity is the product of the upper bounds for the number of steps each nested loop is executed.

Step 5. Construction of the visit-sequences Input: Acyclic graphs $T D P_{p}$ as computed in step 4 , and the vector $P A R T$ as com-
puted in step 3 .

Output: A visit-sequence for each $p \in P$. Method: Starting from an empty graph $V S_{p}$ over $A V_{p}$ arcs are added according
to Definition 9 .

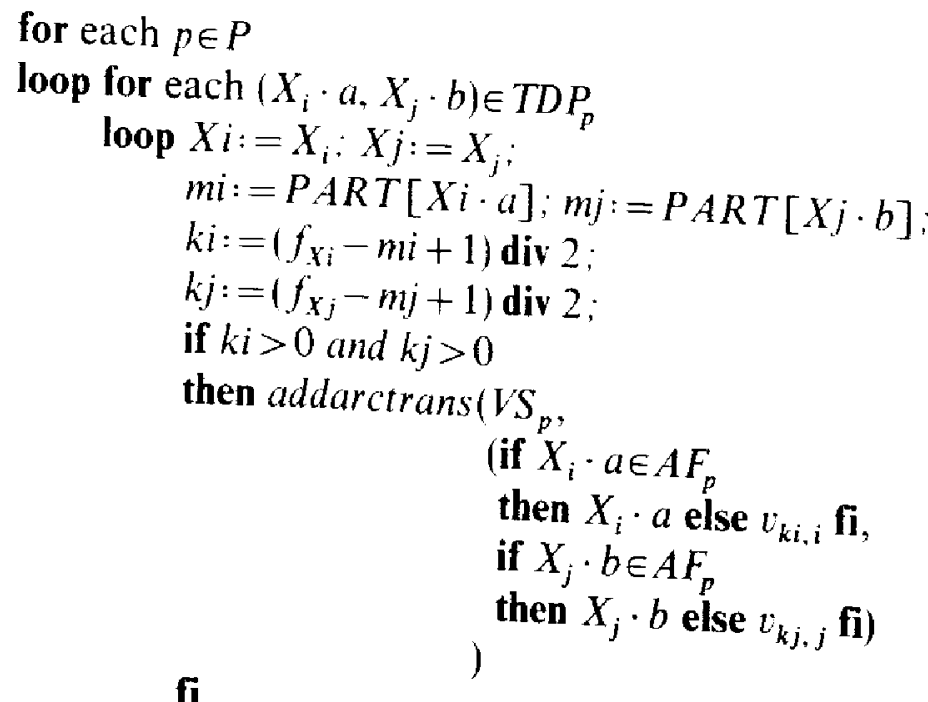

repeat; 
(* add arcs until $V S_{p}$ is linearly ordered *)

for each $g \in A V_{p}$

loop for each $h \in A V_{p}$

loop if $(g, h),(h, g) \notin V S_{p}$

then

if $g=v_{k, 0}$ and $k=n v_{X}, X=X_{0}$

then addarctrans $(h, g)$

else addarctrans $(g, h)$

fi

fi

repeat

repeat

repeat

Complexity: $\mathrm{O}\left(|P||D|^{4}\right)$

For the two loops on the second level $|D|^{2}$ is an upper bound for the arcs added by addarctrans.

The complexity of the whole algorithm is given by the following sum:

$$
\begin{aligned}
& \text { Step 1: } O\left(|P||D|^{4}\right)+ \\
& \text { Step 2: } O\left(|G||X|^{2}|D|^{2}\right)+ \\
& \text { Step 3: } O\left(|V||X|^{3}\right)+ \\
& \text { Step 4: } O\left(|P||R||X|^{2}|D|^{2}\right)+ \\
& \text { Step 5: } O\left(|P||D|^{4}\right)
\end{aligned}
$$

Since $|G| \geqq|V|$ step 3 does not contribute to the total complexity. The items for step 2 and 4 are equal, because $|G|=|P||R|$. Hence the formula reduces to

$$
\begin{aligned}
& \mathrm{O}\left(|P||D|^{4}+|G||X|^{2}|D|^{2}\right)= \\
& \mathrm{O}\left(|X|^{4}|G|\left(|R|^{3}+|R|^{2}\right)\right)= \\
& \mathrm{O}\left(|X|^{4}|G||R|^{3}\right)
\end{aligned}
$$

The length of the context-free grammar contributes only linearly to the total complexity. The maximum length $|R|$ of the syntactic rules is usually bound by a small constant. So the most significant item is $|X|^{4}$ the maximum number of attributes associated to a single symbol. For AGs defining programming languages this number will be rather small, too. Thus the computation of transitive closures can be implemented by more powerful set operations, which will reduce the complexity to $\mathrm{O}\left(|X|^{3}|G||R|^{2}\right)$. An implementation of this algorithm on a SIEMENS 7760 needed about 60 seconds for the analysis of an $A G$ for the rather large language PEARL $(|X|=25,|G|=849,|R|=8)$.

\section{Conclusion}

In this paper a new class of attributed grammars is introduced: ordered attributed grammars. The expressive power of ordered attributed grammars is larger than that of any other subclass of attributed grammars based on a fixed evaluation strategy. It is sufficiently large for the definition of programming languages. 
The class is well suited for both programming language definition and automatic compiler generation. The definition is based on the natural concept of linearly ordered dependencies between the attributes of syntactic units. Therefore a language designer can define the context dependent properties of the language statically, without considering any (predefined) evaluation strategy. The evaluation algorithm can be implemented in a simple and efficient way. It is parameterized by local evaluation rules, which are computed from the given attributed grammar. This principle is comparable to well known and widely used techniques for parser generation (e.g. LALR(1)-technique). In [7] it is shown how this method can be integrated in a compiler generating system based on attributed
grammars.

Acknowledgements. I am indebted to G. Goos and my colleagues for many stimulating discussions, to R. Loos for his valuable remarks on the complexity analysis, and to W.M. Waite for his comments
on the manuscript.

\section{References}

1. Bochmann, G.V.: Semantic evaluation from left to right. CACM 19,55-62 (1976) 2. Ganzinger, H., Ripken, K., Wilhelm, R.: MUG1 - an incremental compiler-compiler. In: Proc. of
ACM 1976 Ann. Conf., pp. 535-540, 1976 3. Giegerich, R., Wilhelm, R.: Implementierbarkeit attributierter Grammatiken. In: Informatik
Fachberichte 10, pp. 17-36, 1977

4. Jazayeri, M., Ogden, W.F., Rounds, W.C.: The intrinsically exponential complexity of the circu-

larity problem for attributed grammars. CACM 18,679-706 (1975)
5. Jazayeri. M., Walter, K.G.: Alternating semantic evaluator. pp. $230-234,1975$

6. Kastens, U.: Ein Übersetzer-erzeugendes System auf der Basis attributierter Grammatiken. Fak.f.

7. Kastens, U. A A ADIN Karlsruhe, Interner Bericht 10, 1976

matik. Universität Karlsruhe Ine Definitionssprache für attributierte Grammatiken. Fak. I. Infor-

8. Kennedy, K.. Warren, S.K. Anterner Bericht 7, 1979

Conference record of the $3^{\text {rd }}$ ACMatic generation of efficient evaluators for attribute grammars. 1976

9. Knuth, D.E.: Semantics of context-free languages. Math. Syst. Theory 2, 127-145 (1968)

10. Knuth. D.E. Semantics of context-free languages: correction. Math. Syst. Theory 5, 95-96 (1971) tion Processing 74.1974. G.: A (truly) usable and portable compiler writing system. In: Informa-

LILA, an introduction. In: Huens, J., Huybrechts, M.: Project LILA: The ELL(1) generator of 1977

13. Lewis, P.M. Rosenkrantz, D.J., Stearns. R.E. Aitributed transtations. Journal of Computer and System Science 9, 279-307 11974)

14. Lorho, B.: Semantic autributes language Implementation pp processing in the system DELTA. In: Methods of Algorithmic

15. Räihä, K.-J.: On attribute pp. 21-40. Springer Verlag 1977

University of Helsinki, Dep. of Comp. Sc 1977 use in a compiler writing system. Rep. A-1977-4,

16. Schulz, W.A.: Semantic analysis and 1977

Colorado, Dep. of Comp. Sc., PhD thesis target language synthesis in a translator, University of

17. Wilhelm. R.: Baumtransformatoremesis 1976

Implementierung. TU München, Bericht, pp. $77-13$, mit Baumtransduktoren und Aspekte der

Received September 21, 1978 ; July 20,1979 October 5,1979 